\title{
AN INFINITE-DIMENSIONAL VERSION OF LIAPUNOV'S CONVEXITY THEOREM
}

\section{Vladimir Drobot}

The classical theorem of Liapunov asserts that the range of a finite measure with values in a finite-dimensional vector space is convex and closed (see [1], [2], [3], [4]). In his later paper [5], Liapunov gives an example of an $L_{1}$-valued measure whose range is compact but not convex. In this note, we prove a weaker version of Liapunov's theorem, where the measure takes values in a Hilbert space and is absolutely continuous with respect to a numerical measure.

Let $(\mathrm{S}, \mathscr{F}, \mu)$ denote a measure space, where $\mu$ is a positive, nonatomic measure with $\mu(S)=1$, and let $\mathrm{H}$ denote a real Hilbert space with the inner product $(\mathrm{x}, \mathrm{y})$ and norm $\|\mathrm{x}\|$.

THEOREM. Let $\mathrm{f}: \mathrm{S} \rightarrow \mathrm{H}$ be an integrable function (that is, $\left.\int\|\mathrm{f}\| \mathrm{d} \mu<\infty\right)$, and let $\mathrm{R}=\mathrm{R}(\mathrm{f})$ be the set of all vectors of the form $\int_{\mathrm{E}} \mathrm{fd} \mu(\mathrm{E} \in \mathscr{F})$. Then $\overline{\mathrm{R}}$ is convex.

The proof is motivated by a method due to Halkin [2] who considered the finitedimensional case only. We need several lemmas.

LEMMA 1. Let $\left\{\mathrm{x}_{1}^{\prime}, \mathrm{x}_{2}^{\prime}, \cdots, \mathrm{x}_{\mathrm{N}}^{\prime}\right\}$ be a collection of $\mathrm{N}$ vectors in $\mathrm{H}$ such that $\sum \mathrm{x}_{\mathrm{i}}^{\prime}=0$. Then the $\mathrm{x}_{\mathrm{j}}^{\prime}$ can be rearranged to form a set $\left\{\mathrm{x}_{1}, \mathrm{x}_{2}, \cdots, \mathrm{x}_{\mathrm{N}}\right\}$ such that

$$
\left\|\sum_{i=1}^{n} x_{i}\right\|^{2} \leq \sum_{i=1}^{N}\left\|x_{i}\right\|^{2} \quad(1 \leq n \leq N)
$$

Proof. We choose $\mathrm{x}_{1}$ arbitrarily. Having chosen $\mathrm{x}_{1}, \mathrm{x}_{2}, \cdots, \mathrm{x}_{\mathrm{n}}$, we select $x_{n+1}$ to be one of the remaining vectors with the property that

$$
\left(\mathrm{x}_{1}+\mathrm{x}_{2}+\cdots+\mathrm{x}_{\mathrm{n}}, \mathrm{x}_{\mathrm{n}+1}\right) \leq 0
$$

Such a choice is always possible, because

$$
0=\left(\sum_{1}^{N} x_{i}^{\prime}, \sum_{1}^{N} x_{i}^{\prime}\right)=\left(\sum_{1}^{n} x_{i}, \sum_{1}^{n} x_{i}\right)+2 \sum_{j=n+1}^{N}\left(\sum_{1}^{n} x_{i}, x_{j}^{\prime}\right)+\left(\sum_{n+1}^{N} x_{j}^{\prime}, \sum_{n+1}^{N} x_{j}^{\prime}\right) .
$$

Since the first and the last inner products are nonnegative, at least one summand in the middle term must be nonpositive. Our arrangement of the $x_{j}$ gives us the equations 


$$
\left\|\sum_{1}^{n+1} x_{i}\right\|^{2}=\left(\sum_{1}^{n+1} x_{i}, \sum_{1}^{n+1} x_{i}\right)=\left\|\sum_{1}^{n} x_{i}\right\|^{2}+\left\|x_{n+1}\right\|^{2}+2\left(\sum_{1}^{n} x_{i}, x_{n+1}\right) .
$$

The result now follows by induction.

The following lemma was proved by P. R. Halmos [3]. that

LEMMA 2. For every set $\mathrm{E} \in \mathscr{F}$, there exists a function $\phi: \mathrm{E} \rightarrow[0,1]$ such

$$
\mu(\{\mathrm{x} \in \mathrm{E}: \phi(\mathrm{x})<\lambda\})=\lambda \mu(\mathrm{E}) .
$$

The next result is crucial.

LEMMA 3. Let $\mathrm{g}: \mathrm{X} \rightarrow \mathrm{H}$ be an integrable function (that is, $\int\|\mathrm{g}\| \mathrm{d} \mu<\infty$ ). Then, for every $\varepsilon>0$, there exists a function $\Phi: \mathrm{X} \rightarrow[0,1]$ such that

i) $\left\|\int_{E(\lambda)} \operatorname{gd} \mu-\lambda \int_{S} \mathrm{gd} \mu\right\|<\varepsilon$, where $\mathrm{E}(\lambda)=\{\mathrm{x}: \Phi(\mathrm{x})<\lambda\}$, and

ii) $\mu(\{\mathrm{x}: \Phi(\mathrm{x})<\lambda\})=\lambda$.

(We denote the collection of such functions $\Phi$ by $\mathrm{K}(\mathrm{g}, \varepsilon)$.)

Proof. We may assume $\int_{\mathrm{S}} \mathrm{g} \mathrm{d} \mu=0$, since otherwise we could apply the result to $g-\int_{S} g d \mu$. Choose an integer $N$ such that if $\mu(E) \leq \frac{1}{N}$, then

$$
\int_{\mathrm{E}}\|\mathrm{g}\| \mathrm{d} \mu<\min \left\{\frac{1}{2} \varepsilon, \frac{1}{4} \varepsilon^{2}\left[\int_{\mathrm{X}}\|\mathrm{g}\| \mathrm{d} \mu\right]^{-1}\right\}=\eta .
$$

Select a function $\phi: \mathrm{X} \rightarrow[0,1]$ as in Lemma 2, so that $\mu\{\mathrm{x}: \phi(\mathrm{x})<\lambda\}=\lambda$. Let

$$
A_{i}^{\prime}=\left\{x: \frac{i-1}{N} \leq \phi(x)<\frac{i}{N}\right\} \quad(i=1,2, \cdots, N) .
$$

Then $\mu\left(\mathrm{A}_{\mathbf{i}}^{\prime}\right)=\frac{1}{\mathrm{~N}}, \sum_{\mathbf{i}} \int_{\mathrm{A}_{i}^{\prime}} \mathrm{g} \mathrm{d} \mu=0$, and $\int_{\mathrm{A}_{\mathbf{i}}^{\prime}}\|\mathrm{g}\| \mathrm{d} \mu<\eta$.

By Lemma $1, A_{i}^{\prime}$ can be rearranged into $\left\{A_{1}, A_{2}, \cdots, A_{N}\right\}$, say, such that

$$
\left\|\sum_{i=1}^{\mathrm{n}} \int_{\mathrm{A}_{i}} \mathrm{gd} \mu\right\|^{2} \leq \sum_{1}^{\mathrm{N}}\left\|\int_{\mathrm{A}_{\mathrm{i}}} \mathrm{gd} \mu\right\|^{2} \leq \sum_{1}^{\mathrm{N}} \eta \int_{\mathrm{A}_{i}}\|\mathrm{~g}\| \mathrm{d} \mu \leq \frac{1}{4} \varepsilon^{2} \quad(1 \leq \mathrm{n} \leq \mathrm{N}) .
$$

Hence each partial sum satisfies the inequality

$$
\left\|\sum_{i=1}^{\mathrm{n}} \int_{\mathrm{A}_{\mathrm{i}}} \mathrm{gd} \mu\right\| \leq \frac{1}{2} \varepsilon .
$$


For each index $i(i=1,2, \cdots, N)$, we choose a function $\phi_{i}: A_{i} \rightarrow[0,1]$ as in Lemma 2. Set

$$
\Phi(x)=\sum_{i=1}^{N} \frac{i-1}{N} I_{A_{i}}(x)+\sum_{i=1}^{N} \frac{1}{N} \phi_{i}(x),
$$

where $I_{F}$ is the characteristic function of $F$ and $\phi_{i}(x)=0$ for $x \notin A_{i}$. Now we can write the set $E(\lambda)$ as

$$
\begin{aligned}
& E(\lambda)=\{\mathrm{x}: \Phi(\mathrm{x})<\lambda\} \\
&=\bigcup_{\frac{\mathrm{i}-1}{\mathrm{~N}} \leq \lambda} \mathrm{A}_{\mathrm{i}} \cup\left\{\mathrm{A}_{[\mathrm{N} \lambda]+1} \cap\{\mathrm{x}: \phi[\mathrm{N} \lambda]+1\right. \\
&
\end{aligned}
$$

where $[\alpha]$ denotes the greatest integer not exceeding $\alpha$.

The sets whose union we take in (3) are disjoint, so that

$$
\left\|\int_{E(\lambda)} g \mathrm{~d} \mu\right\| \leq\left\|\sum_{i=1}^{[\mathrm{N} \lambda]} \int_{\mathrm{A}_{\mathrm{i}}} \mathrm{g} \mathrm{d} \mu\right\|+\int_{\mathrm{A}_{[\mathrm{N} \lambda]+1}}\|\mathrm{~g}\| \mathrm{d} \mu .
$$

The first partial sum is less than $\varepsilon / 2$, by (2). The integral is less than $\varepsilon / 2$, by (1). The result now follows.

We proceed to prove the theorem. It is enough to show that if $\mathbf{E}$ and $\mathbf{F}$ are two measurable subsets of $\mathrm{X}$, then for every $\lambda \in[0,1]$ and every $\varepsilon>0$, there exists a measurable set $C(\lambda)$ such that

$$
\left\|\int_{C(\lambda)} \mathrm{fd} \mu-\lambda \int_{E} \mathrm{fd} \mu-(1-\lambda) \int_{F} \mathrm{fd} \mu\right\|<\varepsilon .
$$

We select $\Phi \in \mathrm{K}\left(\mathrm{fI}_{\mathrm{E}-\mathrm{F}}, \varepsilon / 2\right)$ and $\psi \in \mathrm{K}\left(\mathrm{fI}_{\mathrm{F}-\mathrm{E}}, \varepsilon / 2\right)$ (the sets $\mathrm{K}$ are defined by Lemma 3) and put

$$
\mathbf{C}(\lambda)=\{\mathrm{E} \cap \mathrm{F}\} \cup\{\mathrm{x} \in \mathbf{E}-\mathrm{F}: \Phi(\mathrm{x})<\lambda\} \cup\{\mathrm{x} \in \mathbf{F}-\mathrm{E}: \psi(\mathrm{x})<1-\lambda\} .
$$

Since the sets above are disjoint, we obtain the inequalities

$$
\begin{aligned}
\| \int_{C(\lambda)}-\lambda\left[\int_{E \cap F}+\int_{E-F}\right] & +(1-\lambda)\left[\int_{E \cap F}+\int_{F-E}\right] \| \\
\leq & \left\|\int_{\{\Phi<\lambda\}} f I_{E-F} d \mu-\lambda \int f I_{E-F} d \mu\right\| \\
& +\left\|\int_{\{\psi<1-\lambda\}} f I_{F-E} d \mu+(1-\lambda) \int f I_{F-E} d \mu\right\|<\varepsilon .
\end{aligned}
$$

This completes the proof. 


\section{REFERENCES}

1. D. Blackwell, The range of certain vector integrals. Proc. Amer. Math. Soc. 2 (1951), 390-395.

2. H. Halkin, Some further generalizations of a theorem of Lyapounov. Arch. Rational Mech. Anal. 17 (1964), 272-277.

3. P. R. Halmos, The range of a vector measure. Bull. Amer. Math. Soc. 54 (1948), 416-421.

4. A. A. Lyapunov, Sur les fonctions-vecteurs complètement additives. (Russian. French summary) Bull. Acad. Sci. URSS. Sér. Math. [Izvestia Akad. Nauk SSSR] 4 (1940), 465-478.

5. - Sur les fonctions-vecteurs complètement additives. (Russian. French summary) Bull. Acad. Sci. URSS. Sér. Math. [Izvestia Akad. Nauk SSSR] 10 (1946), 277-279.

State University of New York at Buffalo

Buffalo, New York 14226 